\title{
Microscopia eletrônica de transmissão da liga Ti-10Mo-20Nb envelhecida após forjamento a quente
}

\author{
Transmission electron microscopy of aged Ti-10Mo-20Nb alloy after \\ hot swaging
}

\author{
${ }^{1}$ Sinara Borborema Gabriel \\ ${ }^{2}$ Renato Baldan \\ 3 Juliana Torres \\ ${ }^{4}$ Carlos Angelo Nunes \\ ${ }^{5}$ Paulo Roberto Mei \\ ${ }^{6}$ Nathalia Rodrigues Oliveira
}

1 Doutora em Engenharia Metalúrgica e de Materiais pela Universidade Federal do Rio de Janeiro - COPPE/UFRJ / sinarab@msn.com
2 Doutor em Engenharia de Materiais pela Universidade de São Paulo - EEL/USP - Lorena, São Paulo / renatobaldan@gmail.com
3 Graduada em Engenharia de Produção pelo Centro Universitário de Volta Redonda - UniFOA / juliana_torres_5@hotmail.com
4 Doutor em Engenharia Mecânica pela Universidade Estadual de Campinas - UNICAMP - Campinas / cnunes@demar.eel.usp.br
5 Doutor em Engenharia Mecânica pela Universidade Estadual de Campinas - UNICAMP - Campinas / pmei@fem.unicamp.br
6 Graduada em Engenharia de Produção pelo Centro Universitário de Volta Redonda - UniFOA / nathalia_roliveira@yahoo.com.br

\section{RESUMO}

Ligas de Ti são muito utilizadas em aplicações biomédicas. Dentro dessa classe, as ligas de Ti do tipo $\beta$ metaestável se destacam, pois por meio de processamentos termomecânicos é possível obter propriedades mecânicas e, em especial, um módulo de elasticidade adequado para aplicação biomédica. Essas ligas, para serem usadas em aplicações ortopédicas, requerem um balanço entre alta resistência mecânica e baixo módulo de elasticidade, a fim de se evitar o efeito "stress shielding" Estudos preliminares mostraram que a microestrutura da liga Ti-10Mo-20Nb, após forjamento a frio e envelhecimento a $500^{\circ} \mathrm{C} / 24 \mathrm{~h}$, apresentou uma distribuição bimodal da fase a na matriz $\beta$. $O$ objetivo deste trabalho foi caracterizar microestruturalmente a liga Ti-10Mo-20Nb na condição envelhecida a $500^{\circ} \mathrm{C}$ por $24 \mathrm{~h}$, após forjamento a quente. A caracterização microestrutural consistiu em análises por difração de raios $X$ e microscopia eletrônica de transmissão. De acordo com os resultados obtidos, enquanto o forjamento a frio acarretou numa distribuição bimodal da fase alfa na matriz beta, o forjamento a quente acarretou numa precipitação fina e homogênea da fase alfa na matriz beta.

\section{PALAVRAS-CHAVE}

Ligas de Ti; microestrutura; biomateriais; processamento.

\section{ABSTRACT}

Ti alloys are widely used in biomedical applications. Within this class, metastable $\beta$-Ti alloys stand, because through thermomechanical processing it is possible to obtain mechanical properties and in particular one suitable Young's modulus for biomedical applications. These alloys require high mechanical strength and a low Young's modulus to avoid stress shielding. Preliminary studies showed that the microstructure of the Ti-10Mo$20 \mathrm{Nb}$ alloy after cold forging and aging $500^{\circ} \mathrm{C} / 24 \mathrm{~h}$ consisted in bimodal distribution of a phase in the $\beta$ matrix. The aim of this study was to characterize the microstructure of Ti-10Mo-20Nb alloy after hot forging and aging at $500^{\circ} \mathrm{C}$ for 24 hours. Microstructural characterization consisted of analyzes by $X$-ray diffraction and transmission electron microscopy. According to the results, while the cold forging resulted in a bimodal a distribution in the $\beta$ matrix, hot forging resulted in a thin and homogeneous a precipitation in the $\beta$ matrix.

\section{Como você deve citar?}

GABRIEL, S. B., et al. Microscopia eletrônica de transmissão da liga Ti-10Mo-20Nb envelhecida após forjamento a quente. Cadernos UniFOA, Volta Redonda, $n^{\circ}$ 25, quadrimestral, p. 45-50, ago. 2014. 


\section{INTRODUÇÃO}

Titânio (Ti) e suas ligas são amplamente usados como implantes ortopédicos por apresentarem boa resistência à corrosão, biocompatibilidade, maior resistência e menor módulo de elasticidade que outros biomateriais metálicos, tais como aço inoxidável e ligas a base de Co-Cr (ZHOU et al., 2005). A liga de Ti mais utilizada em aplicações ortopédicas é a Ti-6Al-4V. Embora essa liga apresente um menor módulo de elasticidade, quando comparada com o aço inoxidável e as ligas de Co-Cr, esse valor (110 - $120 \mathrm{GPa}$ ) é alto comparado ao do tecido ósseo ( 10 - $40 \mathrm{GPa})$. A diferença entre o módulo do metal e do tecido ósseo pode resultar em reabsorção óssea e eventual falha do implante (MAJUMDAR et al., 2008). Além disso, estudos dessa particular liga têm mostrado que a liberação de pequenas quantidades dos elementos $\mathrm{V}$ e Al no corpo humano podem induzir efeitos citotóxicos e desordens neurológicas, respectivamente (RAABE et al., 2007).

Devido a isso, consideráveis esforços têm sido feitos para explorar novas ligas de Ti compostas de elementos não tóxicos, visando aplicações ortopédicas que reúnam as seguintes propriedades: baixo módulo de elasticidade, excelente resistência mecânica, boa resistência à corrosão, fácil conformabilidade e resistência ao desgaste. A fase $\beta$, presente nas ligas de titânio, exibe um menor módulo de elasticidade que as fases a e $a+\beta$ e, também, satisfazem a maior parte dos requisitos para uma bio-liga ideal (KUMAR \& NARAYANAN, 2008, LI et al., 2004, LI et al., 2008, NAG et al., 2007, XU et al., 2006). Vários trabalhos na literatura vêm desenvolvendo ligas do tipo $\beta$ compostas de elementos não tóxicos (BANERJEE et al., 2005, BAPTISTA et al., 2004, BOEHLERT et al., 2005, CREMASCO et al., 2008, GABRIEL et al. 2008, GABRIEL, 2008, GABRIEL et al, 2013, GORDIN et al., 2005, HO et al., 1999, KURODA et al., 1998, Ll et al., 2004, NIINOMl et al., 2005, TANE et al., 2008, XU et al., 2006)

Os estudos preliminares realizados por Gabriel (2008) no processamento termomecânico (forjamento a frio) da liga Ti-10Mo-20Nb mostraram que esta apresenta potencial para aplicação biomédica. Porém, devido à liga apresentar uma alta resistência à deformação a frio, o forjamento a frio se torna uma rota inviável e, por isso, faz-se necessário um estudo mais abrangente, no qual a rota de conformação adotada seja o forjamento a quente.

Nesse contexto, o objetivo deste trabalho foi caracterizar microestruturalmente a liga, na condição envelhecida a $500^{\circ} \mathrm{C}$ por $24 \mathrm{~h}$ após forjamento a quente, de forma a poder comparar os resultados microestruturais com os obtidos pelo forjamento a frio na mesma condição.

\section{PROCEDIMENTO EXPERIMENTAL}

A liga Ti-10Mo-20Nb foi preparada a partir de $\mathrm{Ti}$, Mo e $\mathrm{Nb}$ de pureza comercial por fusão a arco com eletrodo não consumível de tungstênio em atmosfera de argônio. 0 lingote obtido foi tratado a $1000^{\circ} \mathrm{C}$ por $24 \mathrm{~h}$, em um forno tubular com resfriamento em água à temperatura ambiente e, então, forjado a quente $\left(900-1000{ }^{\circ} \mathrm{C}\right)$ até redução em área de $~ 80 \%$. Em seguida, a liga foi envelhecida na temperatura de $500^{\circ} \mathrm{C}$ por $24 \mathrm{~h}$ em um forno tubular com resfriamento em água à temperatura ambiente.

As análises de fases da liga envelhecida na temperatura de $500{ }^{\circ} \mathrm{C}$ por $24 \mathrm{~h}$ (na forma polida) foram realizadas por difração de raios-X (DRX) usando uma Shimadzu modelo DRX 6000, nas seguintes condições: radiação CuKa $(\lambda=1,5418 \AA$ ) com monocromador de grafite, tensão de $40 \mathrm{kV}$, corrente de $30 \mathrm{~mA}$, varredura $(2 \Theta)$ de 30 a 90 graus, passo angular de $0,05^{\circ}$ e tempo de contagem de 5 s por ponto. As fases foram identificadas por meio da comparação com difratograma simulado. As simulações foram realizadas no programa Powdercell (Kraus \& Nolze, 1996), inserindo-se dados das fases b e a (VILLARS \& CALVERT, 1991), como grupo espacial, parâmetros de rede e posições atômicas. 
Além disso, a liga foi caracterizada por microscopia eletrônica de transmissão. Para obtenção das amostras, foi utilizado o afinamento eletrolítico, nas seguintes condições: temperatura de $-20^{\circ} \mathrm{C}$, tensão aplicada de $35 \mathrm{~V}$, com eletrólito composto por $10 \%$ de ácido perclórico e $90 \%$ de metanol. As imagens foram obtidas em um Microscópio Eletrônico de Transmissão marca CM-20 PHILIPS com EDS.

\section{RESULTADOS E DISCUSSÃO}

A Figura 1 mostra o padrão de DRX da liga Ti-10Mo-20Nb, após o envelhecimento a $500^{\circ} \mathrm{C} / 24 \mathrm{~h}$. Verificou-se a presença de reflexões da fase a na matriz $\beta$.

Figura 1 - Difratograma da liga Ti-10Mo-20Nb forjada a quente e envelhecida - $500^{\circ} \mathrm{C} / 24 \mathrm{~h}$.

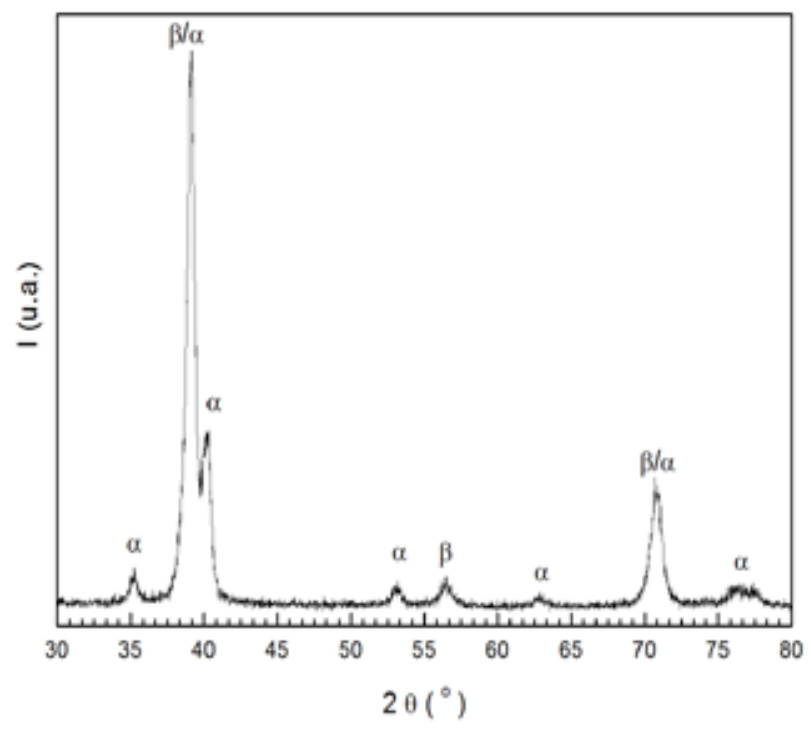

Fonte: (do próprio autor)

A Figura 2 apresenta uma imagem de campo claro da liga forjada a quente e envelhecida a $500^{\circ} \mathrm{C} / 24 \mathrm{~h}$, mostrando finas lamelas da fase a (branca) na matriz $\beta$. Comparando os resultados com os obtidos para a liga cuja rota utilizada foi o forjamento a frio (GABRIEL, 2008), verificou-se que, enquanto o forjamento a quente acarretou na precipitação de finas lamelas da fase a distribuídas homogeneamente na matriz $\beta$, o forjamento a frio acarretou numa precipitação bimodal (partículas lamelares e massivas) da fase alfa na matriz $\beta$. Essa diferença observada nas duas rotas se deve, provavelmente, à precipitação 
da fase $\omega$ na liga forjada a quente, pois, de acordo com a literatura, ela atua como sítios de nucleação para a precipitação da fase a de forma fina e homogênea (IVASISHIN et al, 2005, GABRIEL et al., 2013).

Figura 2 - Análise por MET da liga Ti-10Mo-20Nb envelhecida a $500{ }^{\circ} \mathrm{C} / 24 \mathrm{~h}$ mostrando uma imagem de campo claro da fase a (lamelas brancas) em uma matriz $\beta$.

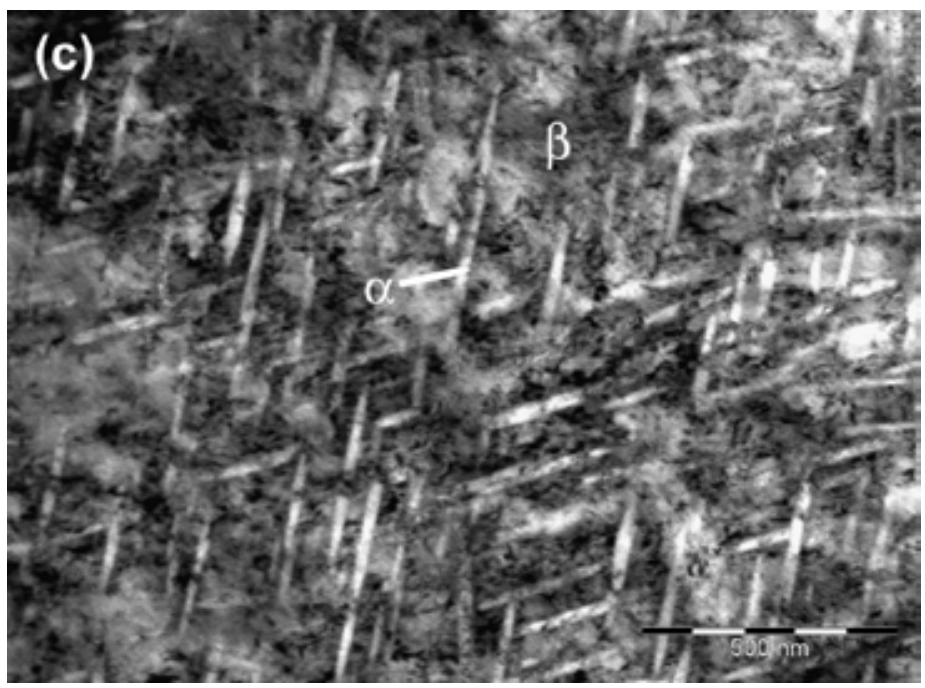

Fonte: (do próprio autor)

Como as propriedades do material é dependente da microestrutura e que, de acordo com Matsumoto et al. (2007), a precipitação da fase a de forma fina e uniforme acarreta no aumento tanto da resistência mecânica quanto do módulo de elasticidade da liga,sugere-se que o forjamento a quente tenha acarretado numa maior dureza da liga e também no aumento do módulo de elasticidade.

\section{CONCLUSÃO}

De acordo com os resultados obtidos para a liga Ti-10Mo-20Nb envelhecida, o forjamento a quente acarretou numa precipitação fina e homogênea da fase alfa na matriz beta, enquanto o forjamento a frio acarretou numa distribuição bimodal da fase alfa na matriz beta. Essa diferença observada nas duas rotas se deve, provavelmente, à precipitação da fase $\omega$ na liga forjada a quente, pois de acordo com a literatura, essa fase atua como sítios de nucleação para a precipitação da fase a de forma fina e homogênea.

\section{AGRADECIMENTO}

Os autosres agradecem ao Departamento de Engenharia de Materiais da Escola de Engenharia de Lorena (EEL/USP), à Universidade Federal do Rio de Janeiro (COPPE/UFRJ) e ao NUPE/UniFOA, pelo desenvolvimento deste trabalho. 


\section{REFERÊNCIAS}

BANERJEE, R., NAG, S., FRASER, H. L., A novel combinatorial approach to the development of beta titanium alloys for orthopaedic implants, Materials Science and Engineering C, v. 25, pp. 282-289, 2005.

BAPTISTA, C. A. R. P., SCHNEIDER, S. G., TADDEI, E. B., et al., Fatigue behavior of arc melted Ti-13Nb13Zr alloy, International Journal of Fatigue, v. 26, pp. 967-973, 2004.

BOEHLERT, C.J., COWEN, C.J., JAEGER, C.R., et al., Tensile and fatigue evaluation of Ti-15Al-33Nb (at. $\%$ ) and Ti-21 Al-29Nb (at.\%) alloys for biomedical applications, Materials Science and Engineering C, v. 25, pp. 263-275, 2005.

CREMASCO, A., OSÓRIO, W. H., FREIRE, C. M. A., et al., Electrochemical corrosion behavior of a Ti-35Nb alloy for medical prostheses, Electrochimica Acta, v. 53, p. 4867-4874, 2008.

GABRIEL, Sinara Borborema. Processamento e caracterização de ligas Ti-Mo-Nb para aplicações biomédicas, 2008, Tese de Doutorado, COPPE/UFRJ, Rio de Janeiro, 2008.

GABRIEL, S. B., NUNES, C. A., SOARES, G. A., 2008, "Production, Microstructural Characterization and Mechanical Properties of Ti-10Mo-xNb alloys", Artificial Organs, v.32, n. 4, pp. 299-304.

GABRIEL, S. B., DE ALMEIDA, L. H., NUNES, C. A., DILLE, J., SOARES, G. A, Maximisation of the ratio of microhardness to the Young's modulus of Ti-12Mo-13Nb alloy through microstructure changes. Materials Science \& Engineering. C, Biomimetic Materials, Sensors and Systems, v.33, pp.3319 - 3324, 2013.

GORDIN, D. M., GLORIANT, T., NEMTOI, G. et al., Synthesis, structure and electrochemical behavior of a beta Ti-12Mo-5Ta alloy as new biomaterial, Materials Letters, v. 59, pp. 2959-2964, 2005.

HO, W. F.; JU, C. P.; CHERN LIN, J. H., Structure and properties of cast binary Ti-Mo alloys", Biomaterials, v. 20, pp. 2115-2122, 1999.

IVASISHIN, O. M., MARKOVSKY, P. E., SEMIATIN, S. L., et al., Aging response of coarse-and fine-grained b titanium alloys, Materials Science and Engineering, v. 405, pp. 296-305, 2005.

KRAUS W. and NOLZE , G. J. Appl. Cryst., v..29, p 301-303, 1996.

KUMAR, S., NARAYANAN, T. S. N. S., Corrosion behavior of Ti-15Mo alloy for dental implant applications", Journal of Dentistry, v. 36, pp. 500-507, 2008.

KURODA, D., NIINOMI, M., MORINAGA, M., et al., Design and mechanical properties of new $b$ type titanium alloys for implant materials, Materials Science and Engineering A, v. 243, pp. 244-249, 1998.

LI, S. J., YANG, R., LI. S., et al., Wear characteristics of Ti-Nb-Ta-Zr and Ti-6Al-4V alloys for biomedical applications, Wear, v. 257, p. 869-876, 2004.

LI, S. J. CUI, T. C., HAO, Y. L., et al., Fatigue properties of a metastable $\beta$-type titanium alloy with reversible phase transformation, Acta Biomaterialia, v. 4, pp. 305-317, 2008. 
MAJUMDAR, P., SINGH, S. B., CHAKRABORTY, M., "Elastic modulus of biomedical titanium alloys by nano-indentation and ultrasonic techniques - A comparative study", Materials Science and Engineering A, v. 489, pp. 419-425, 2008.

MATSUMOTO, H., WATANABE, S., HANADA, S., Microstructures and mechanical properties of metastable $\beta$ TiNbSn alloys cold rolled and heat treated, Journal of Alloys Compounds, v.439, pp. 146-155, 2007.

NAG, S., BANERJEE, R., FRASER, H.L., A novel combinatorial approach for understanding microstructural evolution and its relationship to mechanical properties in metallic biomaterials, Acta Biomaterialia, $\mathrm{v}$. 3, pp. 369-376, 2007.

NIINOMI, M., AKAHORI, T., TAKEUCHI, T., et al., Mechanical properties and cyto-toxicity of new beta type titanium alloy with low melting points for dental applications, Materials Science and Engineering $\mathrm{C}, \mathrm{v}$. 25, pp. 417-425, 2005.

RAABE, D., SANDER, B., FRIÁK, M., et al., Theory-guided botton-up design of $\beta$-titanium alloys as biomaterials based on first principles calculations: Theory and experiments, Acta Materialia, v. 55, p. 4475-4487, 2007.

TANE, M., AKITA, S., NAKANO, T., et al., Peculiar elastic behavior of Ti-Nb-Ta-Zr single crystals, Acta Materialia, v. 56, pp. 2856-2863, 2008.

VILLARS, P.; CALVERT, L.D. Pearson's Handbook of Crystallographic Data for Intermetallic phases, $2^{\circ}$ ed., ASM International, Materials Park, four vol, 1991.

$\mathrm{XU}, \mathrm{W} ., \mathrm{KIM}, \mathrm{K} . \mathrm{B} ., \mathrm{DAS}, \mathrm{J}$, et al., Phase stability its effect on the deformation behavior of Ti-Nb-Ta-In/ Cr b alloys, Scripta Materialia, v. 54, p. 1943-1948, 2006.

ZHOU, Y. L., NIINOMI, M., AKAHORI, T., et al., Corrosion resistance and biocompatibility of Ti-Ta alloys for biomedical applications, Materials Science and Engineering A, v. 398, p. 28-36, 2005. 